\title{
Effect of End Plates on the Surface Pressure Distribution of a Given Cambered Airfoil: Experimental Study
}

\author{
K. S. V. Reddy ${ }^{1 *}$, D. M. Sharma ${ }^{2}$, K. Poddar ${ }^{3}$ \\ Department of Aerospace Engineering, Indian Institute of Technology, Kanpur, 208016 India
}

Email: ksvreddy@iitk.ac.in

\begin{abstract}
While conducting 2-D experiments for flow over airfoil in small wind tunnels, models are usually mounted by spanning the tunnel. However, in large wind tunnels, it is a usual practice to test airfoil models with end plates mounted at its both ends. Thus reduces the blockage, eliminates the tip effects, enables two-dimensional configuration for the flow over airfoil, apart of being cost effective and time saving. Main objective of the present investigation is to study the effect of different sized and shaped end plates on the surface pressure distribution for a given airfoil. Experiments were carried out on a single aerofoil GA(W)-2 at National Wind Tunnel Facility in IIT-Kanpur and surface pressures were measured at constant free stream speed of $30 \mathrm{~m} / \mathrm{s}$ by varying the end-plates shapes at discrete angle of attack $\left(-10^{\circ}\right.$ to $\left.30^{\circ}\right)$. Present study employed three different size end plates A, B, and C. Size of the endplate $\mathrm{A}$ is extended $20 \%$ chord length all along the airfoil cross sectional area. And end plate $B$ is one chord length extended on airfoil except lower surface. In addition end plate B has wedge on the upper side. End plate $\mathrm{C}$ is spanning the tunnel height. These selected varied shaped end-plates had a significant affect on the aerodynamic characteristics along with a considerable variations in the flow phenomena involving flow separations, tip and blockage effects. Results indicates that endplate $\mathrm{C}$ gives the closest 2-D configuration (Figure a). Lift curve slope increases and stall angle decreases as the end plate size increases from $\mathrm{A}$ to $\mathrm{C}$. It appears that for small size end plates (A and B), the stalling angle delayed to higher angles because of the interaction of the outer free stream flow with the flow on the suction side of the airfoil. And this interaction provides the energy to recover pressure and sustains to higher angles at the expense of the lift. And also stalling occurs abruptly. Effect of endplates changes the moment curve slope. Negative slope observed for all the three cases (Figure b).
\end{abstract}
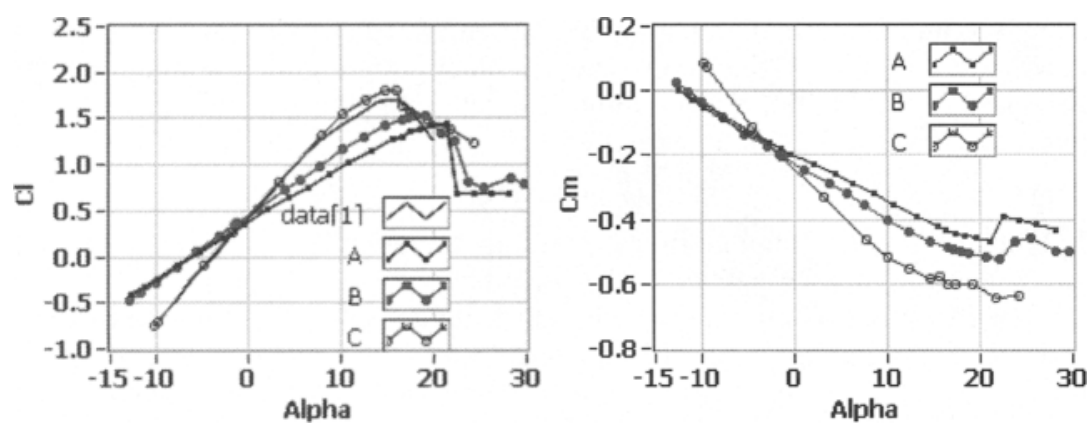

Figure : Effect of endplate size on sectional aerodynamic coefficients verses angle of attack

\section{REFERENCES}

1. McGhee RJ, Beasley WD, Somers DM. Low-speed aerodynamic characteristics of a 13-percent thick airfoil sections designed for general aviation applications. NASA TMX-72697, may 1977 